\title{
A Periodic Microstrip Radial Antenna Array With a Conical Beam
}

\author{
Kuo-Cheng Chen, Yongxi Qian, Senior Member, IEEE, Ching-Kuang C. Tzuang, Fellow, IEEE, and \\ Tatsuo Itoh, Life Fellow, IEEE
}

\begin{abstract}
The analysis and design of a microstrip antenna array with a conical beam are presented. The antenna array employs a periodic structure derived from the microstrip on a perforated ground plane. The microstrip antenna array consists of eight leaky lines evenly distributed around a circle. Each line radiates mainly a pair of forward and backward beams, forming a conical beam. The new leaky line design employs the concept of space-harmonic modulation on the $\mathrm{EH}_{0}$ mode launched at the common joint of the antenna array. The scattering and dispersion characteristics of the leaky line are thoroughly investigated. The fact that the perturbation of the antisymmetric holes made by photolithographic etching excites the leaky $\mathrm{EH}_{1}$ mode is theoretically and experimentally validated. Scattering analyses of the surface currents on the microstrip, however, reveal in detail the physical operation of the leaky line. The dispersion characteristics are, thus, established and shown in a Brillouin diagram, indicating the role of each space harmonic, denoted by its complex propagation constant $\gamma_{m, n}^{ \pm}$• $\gamma_{m, n}^{ \pm}$represents a traveling-wave component of the $n$th higherorder spatial component in association with the $\mathrm{EH}_{m}$ mode: the superscript, $+(-)$, signifies a forward (backward) traveling wave. For the particular leaky line design, $\gamma_{0,-1}^{+}$and $\gamma_{1,0}^{+}$space harmonics, both showing odd-symmetric field polarization, and both outside the triangular Brillouin region, form the backward and forward leaky waves, respectively. When the leaky lines are evenly and collinearly tied, an 11.5-GHz radial antenna array prototype is formed, and emits a conical beam, showing a 3-dB beamwidth of $24^{\circ}$ at the flare angle of $31^{\circ}$.
\end{abstract}

Index Terms-Conical beam, leaky waves, periodic structures, radial antenna array.

\section{INTRODUCTION}

$\mathbf{P}$ ERIODIC structures have recently been extensively studied and developed for use in microwave circuit design [1], [2]. Qian and Itoh reported the propagation characteristics of a uniform microstrip could be altered with noticeable increase in slow-wave factor and a fairly large stopband by simply substituting a ground plane of periodic pattern into a uniform ground plane [2]. The forbidden frequency band is a familiar guiding property of such a periodic structure, and prohibits wave propagation for a certain frequency band. Modifying

Manuscript received October 11, 2000; revised March 11, 2002. This work was supported by the Ministry of Education of Taiwan under Grant 89-E-FA06-2-4.

K.-C. Chen and C.-K. C. Tzuang are with the Institute of Electrical Communication Engineering, National Chiao Tung University, Hsinchu, Taiwan R.O.C. (e-mail: cktzuang@cc.nctu.edu.tw).

Y. Qian is with the Microsemi Corporation, Irvine, CA 92614 USA (e-mail: yongxi.qian@verizon.net).

T. Itoh is with the Electrical Engineering Department, University of California, Los Angeles, CA 90095 USA (e-mail: yqian@ucla.edu; itoh@ee.ucla. edu).

Digital Object Identifier 10.1109/TAP.2003.811064 the ground plane symmetry from even to odd and choosing the appropriate microstrip dimension allows the resultant microstrip to emit leaky waves when launched by a bound, dominant mode $\left(\mathrm{EH}_{0}\right)$ [3]. By radially arranging the leaky line as shown in Fig. 1, a low-profile conical-beam antenna array is presented, manifesting a new alternative approach [4], [5], which is commensurate with common printed-circuit technology. Converting the bound $\mathrm{EH}_{0}$ mode into leaky waves requires no transition to excite the higher order leaky $\mathrm{EH}_{1}$ mode since the leaky line is essentially a distributed mode converter. The inherent mode conversion feature greatly simplifies the power divider/combiner circuit block in Fig. 1. The proposed leaky structure bypasses several transition circuits reported to excite $\mathrm{EH}_{1}$ or $\mathrm{EH}_{2}$ mode. These transition circuits include the asymmetric microstrip step junction with mode suppressor [6], the slotline feed to the micro-slotline [7]-[9], the aperture-coupled feed [10], the broadband microstrip-to-CPS (coplanar strips) transition [11], the micro-CPW line [12], and others.

The rest of this paper is organized as follows. Section II discusses the theory and design of a single leaky line. Section III presents a dispersive Brillouin diagram (k- $\beta$ plot) of the corresponding leaky line. Theoretical data also show that the space harmonics of the Brillouin diagram are a valid approximation of the rigorous electromagnetic field solutions obtained by the full-wave integral equation method. Section IV specifies the complete design of the leaky antenna array, together with measured data. Conclusions are finally made in Section V.

\section{Microstrip ON AN ANTISYMmetricAlly PERForated GROUND PLANE}

Contrary to exciting microstrip leaky modes at higher order by directly connecting certain transition circuits [6]-[12], a distributed mode converter made of a uniform microstrip on a periodically perforated ground plane as shown in Fig. 2 has been developed. The ground plane is patterned by holes etched photolithographically and positioned antisymmetrically with respect to the central cut in the middle of the microstrip. These holes, not necessarily square, are positioned with periodicity $\mathrm{d}_{2}$. With a perimeter of $4 \mathrm{~d}_{1}$, they are small enough to ensure weak modulation on the ground-return currents of the microstrip mode, which may be of type $\mathrm{EH}_{0}$ or $\mathrm{EH}_{1}$. Furthermore, the microstrip is properly designed such that the ideal microstrip (with uniform ground plane) will support the leaky $\mathrm{EH}_{1}$ mode. When a single well-defined microstrip bound $\mathrm{EH}_{0}$ mode is launched at port 1 of Fig. 2, it propagates through the tapered microstrip. The tapering facilitates the necessary impedance transformation from the feed point 


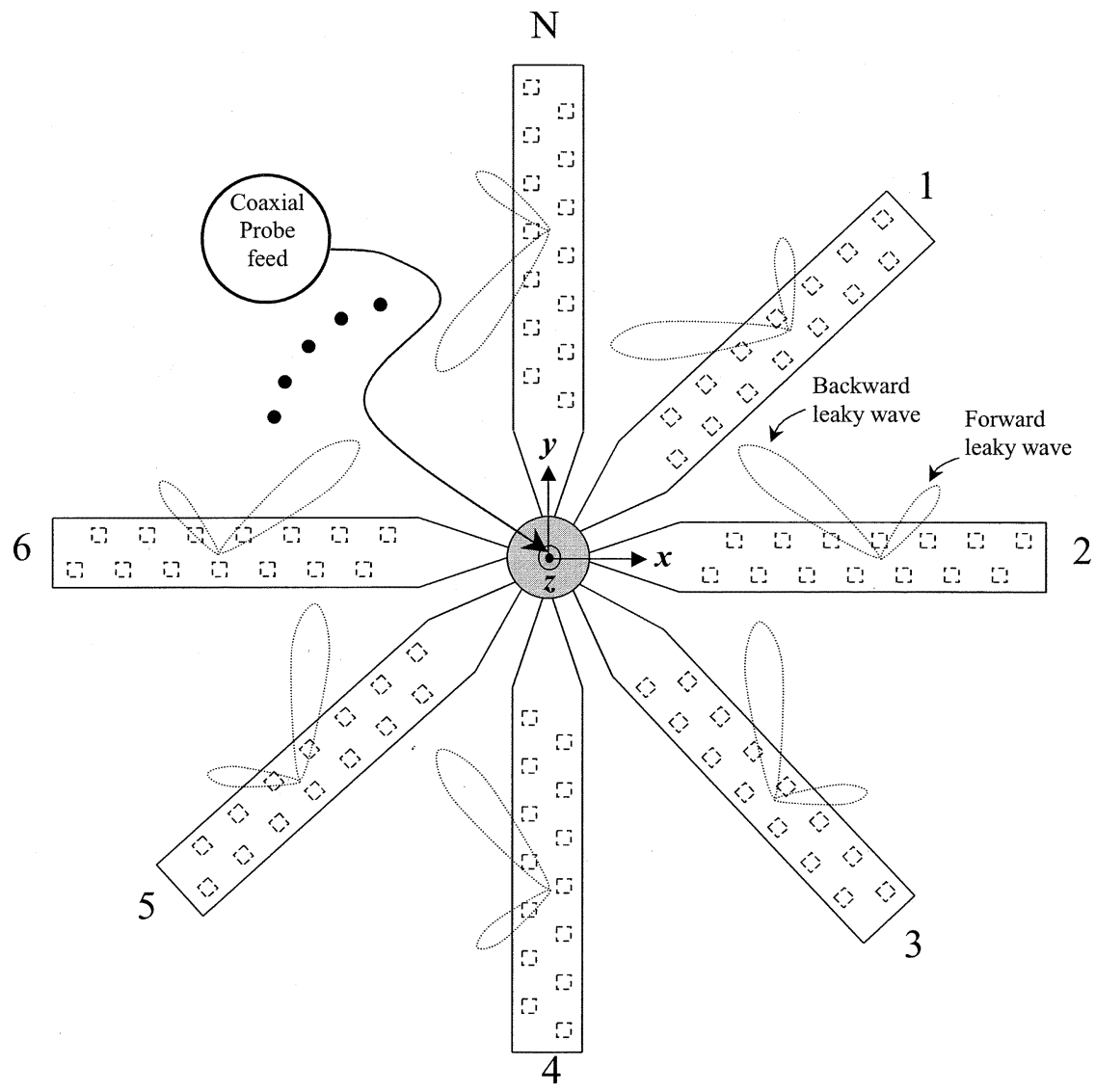

Fig. 1. A generic N-element radial microstrip conical-beam antenna array with periodic perturbations using etched holes on the ground plane.

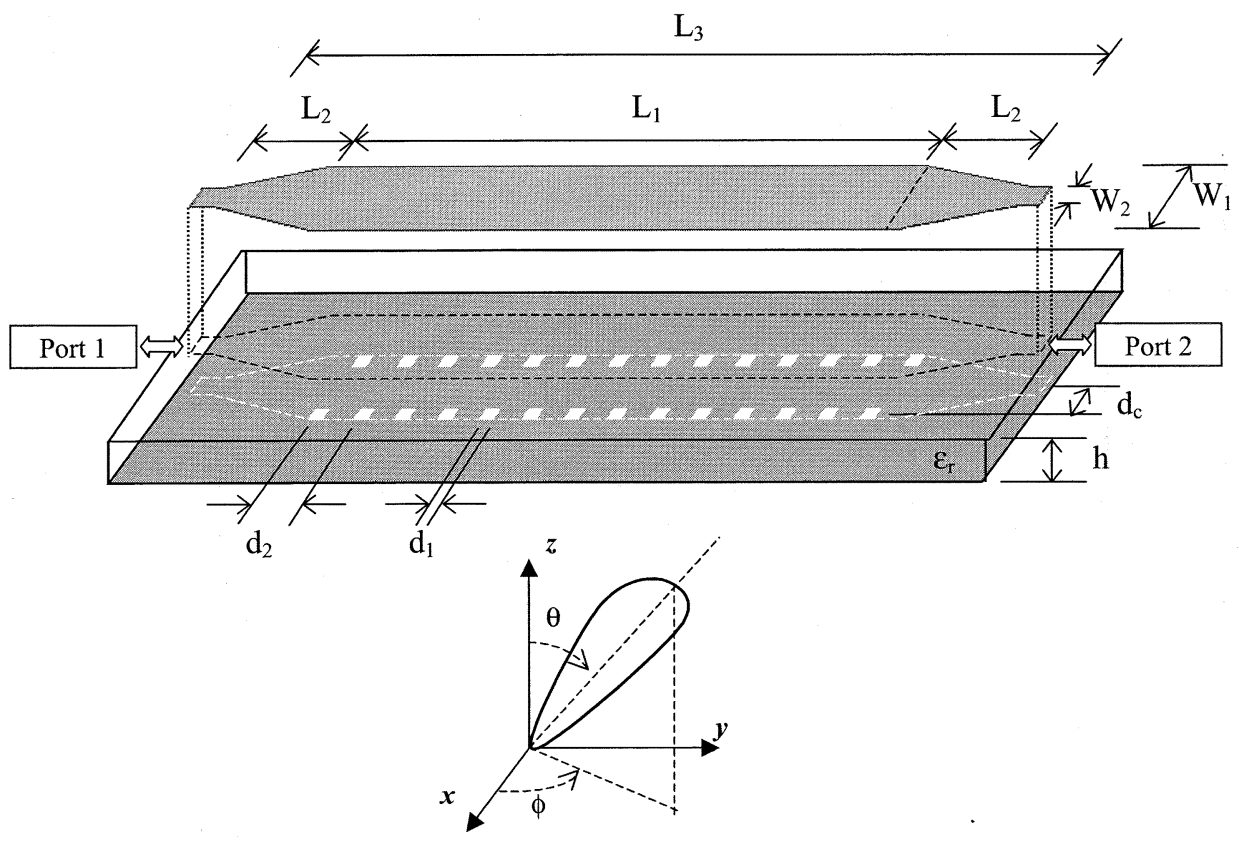

Fig. 2. The structure and spherical coordinates of the leaky line with one or two port(s) attached for evaluation. $\mathrm{L}_{1}=153.8 \mathrm{~mm}, \mathrm{~L}_{2}=14.2 \mathrm{~mm}, \mathrm{~L}_{3}=$ $182.2 \mathrm{~mm}, \mathrm{~d}_{1}=3.0 \mathrm{~mm}, \mathrm{~d}_{2}=12.0 \mathrm{~mm}, \mathrm{~d}_{\mathrm{c}}=3.0 \mathrm{~mm}, \mathrm{~W}_{1}=9.0 \mathrm{~mm}, \mathrm{~W}_{2}=2.0 \mathrm{~mm}, \mathrm{~h}=0.762 \mathrm{~mm}$, and $\varepsilon_{\mathrm{r}}=2.55$.

to each leaky line of lower impedance. At this stage, the propagating mode is still the $\mathrm{EH}_{0}$ mode. When the $\mathrm{EH}_{0}$ mode enters the microstrip (leaky line) with a perforated ground plane that contains antisymmetric holes, it experiences the so-called space-harmonic modulation due to the periodic per- turbations on the modal currents that flow on the ground plane. Section III will present the mathematical details and theoretical results concerning the effects of such periodic modulation on the modal currents. The $\mathrm{EH}_{1}$ leaky mode can be excited because the microstrip structure supports both $\mathrm{EH}_{0}$ 
and $\mathrm{EH}_{1}$ modes and the space-harmonic modulation is of antisymmetric type.

The $\mathrm{EH}_{1}$ mode also experiences the same space-harmonic modulation as that experienced by the launched $\mathrm{EH}_{0}$ mode. The space-harmonic modulations of the $\mathrm{EH}_{0}$ and $\mathrm{EH}_{1}$ modes are carried out simultaneously in a distributed fashion that causes mode conversions, which involve the complicated generation of a variety of space harmonics. These space harmonics are generated in the wider microstrip section of length $\mathrm{L}_{1}$, which in turn behaves like a distributed mode converter. Two tapered microstrip sections are added to the input and output ports to investigate the two-port scattering characteristics and thus explore the guiding characteristics of the mode converter. The length $\mathrm{L}_{2}$ of the linear microstrip taper is sufficient to reduce reflections. Thus, no phasing and matching circuit in the central feeding block is required in Fig. 1. Only the N-way, radial, microstrip combiner/divider [13] is necessary to feed the antenna array.

Following the aforementioned design considerations, the wide microstrip section is $9.0 \mathrm{~mm}$ wide $\left(\mathrm{W}_{1}\right)$ and $153.8 \mathrm{~mm}$ long $\left(\mathrm{L}_{1}\right)$, as depicted in Fig. 2. It is printed on an ULTRALAM 2000 substrate of thickness $0.762 \mathrm{~mm}(\mathrm{~h})$ and relative permittivity $2.55\left(\varepsilon_{r}\right)$. Each of the two columns of the etched square holes on the ground plane has 14 periods. The periodicity of the etched holes is $12.0 \mathrm{~mm}\left(\mathrm{~d}_{2}\right)$. The tapered microstrip section is relatively long with $14.2 \mathrm{~mm}\left(\mathrm{~L}_{2}\right)$. The total length, $\mathrm{L}_{3}$, of the microstrip is $182.2 \mathrm{~mm}$, and is comprised of two tapered sections of length $\mathrm{L}_{2}$, and a wide microstrip of length $\mathrm{L}_{1}$. The width $\left(\mathrm{W}_{2}\right)$ of the feeding microstrip is $2.0 \mathrm{~mm}$. Removing the output taper at port 2 of Fig. 2 forms an open-ended (one-port) leaky line. The coordinate system of Fig. 2 illustrates the leaky line's being positioned along the $y$ axis. The center of the hole to the symmetrical $y-z$ plane is $3.0 \mathrm{~mm}\left(\mathrm{~d}_{\mathrm{c}}\right)$. The length of the sides of each square hole is $3.0 \mathrm{~mm}\left(\mathrm{~d}_{1}\right)$. The size and periodicity of the holes are thus $0.125 \lambda_{\mathrm{o}}$ and $0.5 \lambda_{\mathrm{o}}$, respectively, where $\lambda_{\mathrm{o}}$ is the free-space wavelength of the onset frequency of the $\mathrm{EH}_{1}$ mode at $12.5 \mathrm{GHz}$. The etched pattern on the ground plane of the guiding structure produces weak, periodic perturbations on the modes that propagate alongside the guiding structure.

The inset of Fig. 3 illustrates a uniform microstrip of width $9.0 \mathrm{~mm}(\mathrm{w})$ printed on the same substrate as that mentioned earlier, with thickness $0.762 \mathrm{~mm}(\mathrm{~h})$ and relative permittivity $2.55\left(\varepsilon_{\mathrm{r}}\right)$. The normalized dispersion characteristics of the dominant $\mathrm{EH}_{0}$ mode and the leaky $\mathrm{EH}_{1}$ mode of the ideal microstrip are plotted in Fig. 3. The dispersion characteristics of Fig. 3 are obtained by the two-dimensional (2-D) full-wave integral equation method [7]. The onset frequency of the $\mathrm{EH}_{1}$ mode, at which $\alpha / \mathrm{k}_{0}=0$, is around $12.5 \mathrm{GHz}$. The grayed frequency band, as shown in the abscissa of Fig. 3, highlights the leaky region from 9.4 to $12.5 \mathrm{GHz}$. Notably, at $9.4 \mathrm{GHz}$, two curves defined by $\beta / \mathrm{k}_{0}$ and $\alpha / \mathrm{k}_{0}$ intersect [14]. $\beta / \mathrm{k}_{0}$ and $\alpha / \mathrm{k}_{0}$ are the normalized phase and attenuation constants, respectively. The normalized phase constant of the dominant mode $\left(\mathrm{EH}_{0}\right)$, a bound mode, is nearly constant and close to 1.55 across the 8- to $14-\mathrm{GHz}$ band. By contrast, the leaky $\mathrm{EH}_{1}$ mode shows typical dispersion characteristics: a fast wave of $\beta / \mathrm{k}_{0}$ less than unity with noticeable attenuation in the leaky region.

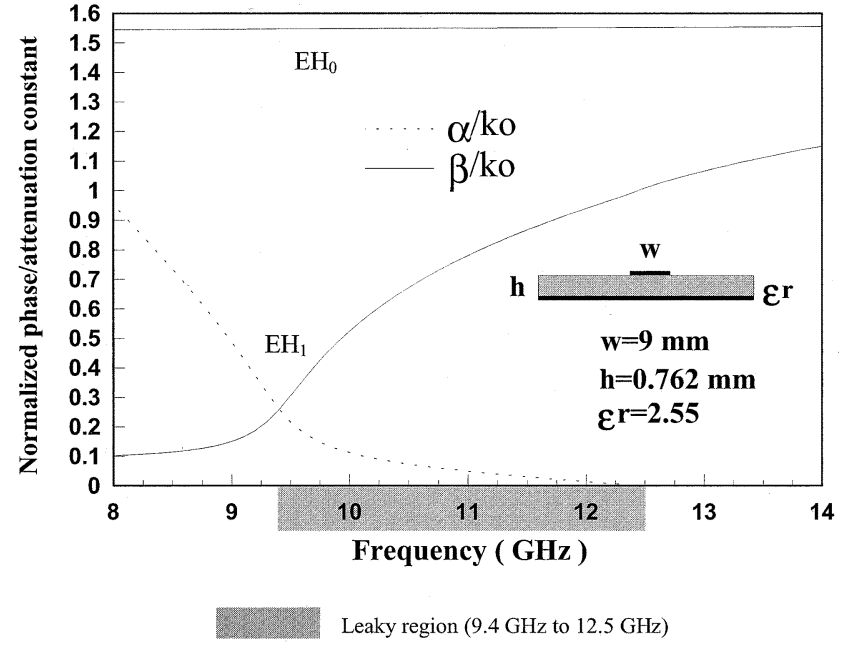

Fig. 3. The dispersion curves of a uniform microstrip line of strip width w. $\left(\mathrm{w}=9.0 \mathrm{~mm}, \mathrm{~h}=0.762 \mathrm{~mm}, \varepsilon_{\mathrm{r}}=2.55\right)$.

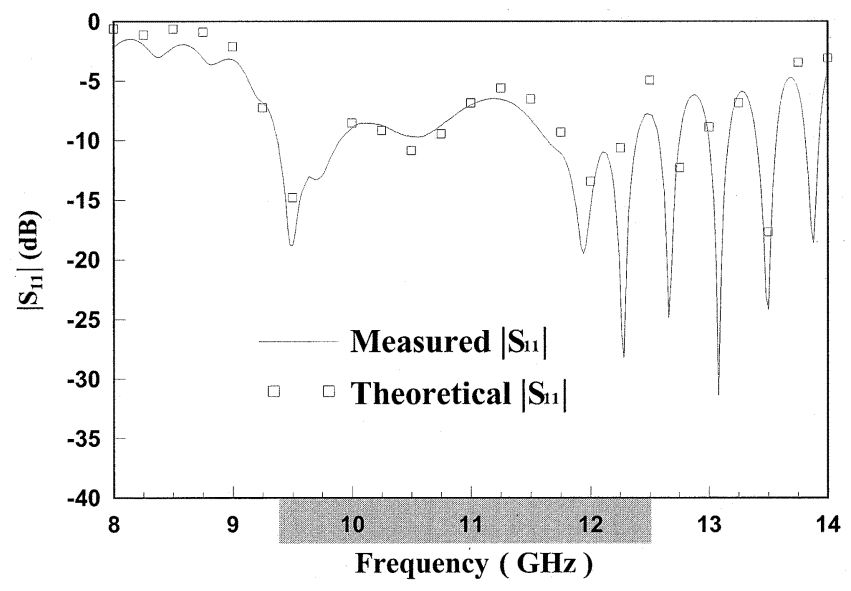

Fig. 4. Measured and theoretical $\left|\mathrm{S}_{11}\right|$ (one-port line) of the single leaky line as shown in Fig. 2.

Fig. 4 indicates that the measured input reflection coefficient $\left(\left|\mathrm{S}_{11}\right|\right)$ (in solid line) closely agree with the theoretical data (in square symbols) applying the full-wave method [15]. $\left|\mathrm{S}_{11}\right|$ is less than $-5 \mathrm{~dB}$ in the leaky region $(9.4-12.5 \mathrm{GHz})$ and beyond, implying that the potentially strong radiating zone covers the entire leaky region of interest and goes beyond the onset frequency of the $\mathrm{EH}_{1}$ mode at $12.5 \mathrm{GHz}$.

Continuing with the two-port measurement of the guiding structure, allows the total losses to be assessed using the relative power absorbed (RPA) value $\left(1-\left|S_{11}\right|^{2}-\left|S_{21}\right|^{2}\right)$. Fig. 5 presents the pertinent measurements. The figure clearly shows three zones of interest. The first is below $9.4 \mathrm{GHz}$, where the proposed structure shows relatively low loss and the value of RPA is between $2 \%$ and $30 \%$ and increases as frequency rises. Most electromagnetic energy propagates through the guiding structure without experiencing large reflections or losses. The second zone lies between 9.4 and $12.5 \mathrm{GHz}$, that is, in the leaky region defined by the $\mathrm{EH}_{1}$ mode in which the RPA value rises sharply from $30 \%$ to $90 \%$, fluctuates, and declines to approximately $60 \%$. Here, the transmission coefficient, $\left|\mathrm{S}_{21}\right|$, drops dramatically to about $-40 \mathrm{~dB}$ and shows good input matching to $50 \Omega$ with $\left|\mathrm{S}_{11}\right|$ between $-6.2 \mathrm{~dB}$ and $-11.8 \mathrm{~dB}$. The third zone 

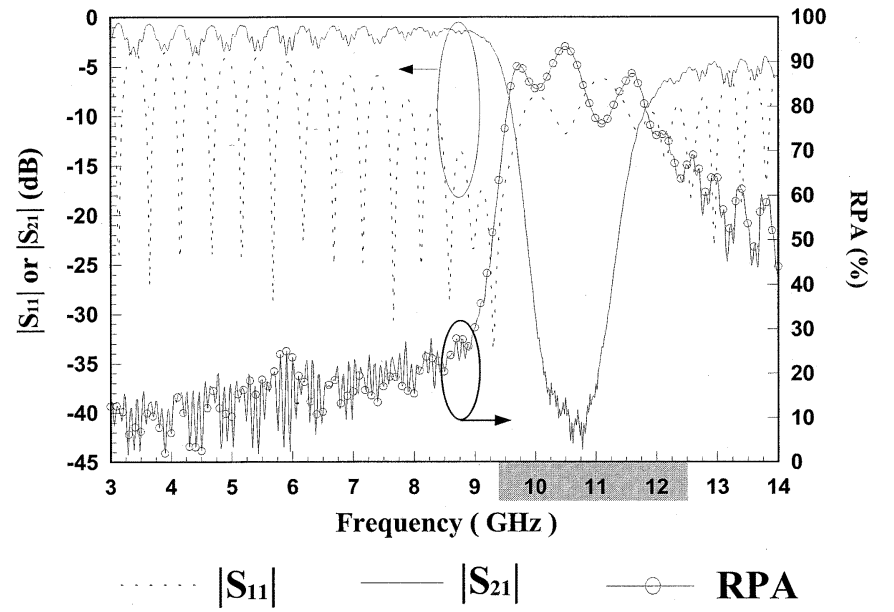

Fig. 5. Measured $\left|\mathrm{S}_{11}\right|,\left|\mathrm{S}_{21}\right|$ and RPA (relative power absorbed, $1-\left|\mathrm{S}_{11}\right|^{2}-$ $\left|\mathrm{S}_{21}\right|^{2}$ ) of the two-port leaky line as shown in Fig. 2.

is above $12.5 \mathrm{GHz}$, where the RPA value fluctuates around $50 \%$. This zone also represents high losses, although the transmission coefficient $\left(\left|\mathrm{S}_{21}\right|\right)$ rises sharply from about $-40 \mathrm{~dB}$ to $-5 \mathrm{~dB}$. The radiation in the third zone is not from the leaky $\mathrm{EH}_{1}$ mode of the uniform microstrip line. The next section will demonstrate how the extended radiation region can be related to the space harmonics.

\section{SPACE HARMONICS AND BRILlOUIN Diagram}

Waves that travel along an axially periodic structure are essentially subject to the electromagnetic field, represented by Floquet's theorem [16], [17]. Given that the proposed guiding structure is periodic, Floquet's theorem can approximate the excited surface currents on the periodic structure by summing an infinite number of traveling-wave components (space harmonics). Since the antisymmetric holes on the ground plane of Fig. 2 spatially modulate the ground-return currents, the space harmonics of the $\mathrm{EH}_{0}$ mode and the higher-order $\mathrm{EH}_{1}$ mode should be included. Therefore, providing the time-harmonic factor $\mathrm{e}^{j \omega t}$ is assumed, the surface current $J_{s}$ on the strip can be represented as

$$
\begin{aligned}
J_{s} \approx & J_{0,0}^{+} e^{-j \gamma_{0,0}^{+} y}+\sum_{n=1}^{\infty} J_{0, n}^{+} e^{-j \gamma_{0, n}^{+} y}+\sum_{n=-1}^{-\infty} J_{0, n}^{+} e^{-j \gamma_{0, n}^{+} y} \\
& +J_{0,0}^{-} e^{-j \gamma_{0,0}^{-} y}+\sum_{n=1}^{\infty} J_{0, n}^{-} e^{-j \gamma_{0, n}^{-} y}+\sum_{n=-1}^{-\infty} J_{0, n}^{-} e^{-j \gamma_{0, n}^{-} y} \\
& +J_{1,0}^{+} e^{-j \gamma_{1,0}^{+} y}+\sum_{n=1}^{\infty} J_{1, n}^{+} e^{-j \gamma_{1, n}^{+} y}+\sum_{n=-1}^{-\infty} J_{1, n}^{+} e^{-j \gamma_{1, n}^{+} y} \\
& +J_{1,0}^{-} e^{-j \gamma_{1,0}^{-} y}+\sum_{n=1}^{\infty} J_{1, n}^{-} e^{-j \gamma_{1, n}^{-} y}+\sum_{n=-1}^{-\infty} J_{1, n}^{-} e^{-j \gamma_{1, n}^{-} y}
\end{aligned}
$$

and

$$
\begin{aligned}
& \gamma_{0, n}^{ \pm}=\beta_{0, n}^{ \pm}-j \alpha_{0, n}^{ \pm}=\left(\beta_{0,0}^{ \pm} \pm \frac{2 \pi}{d_{2}} n\right)-j \alpha_{0, n}^{ \pm} \\
& \gamma_{1, n}^{ \pm}=\beta_{1, n}^{ \pm}-j \alpha_{1, n}^{ \pm}=\left(\beta_{1,0}^{ \pm} \pm \frac{2 \pi}{d_{2}} n\right)-j \alpha_{1, n}^{ \pm}
\end{aligned}
$$

where $\gamma_{0, n}^{ \pm}\left(\gamma_{1, n}^{ \pm}\right)$is the complex propagation constant of the $n$th space harmonic of the dominant $\mathrm{EH}_{0}$ (higher-order $\mathrm{EH}_{1}$ ) mode, propagating along the longitudinal $y$ direction, and $\mathrm{d}_{2}$ is the periodicity of the leaky line. (See Fig. 2.) In (2), $\beta_{p, n}^{ \pm}(p=$ $0,1)$ and $\alpha_{p, n}^{ \pm}(p=0,1)$, respectively, denote the phase and attenuation constants of the $n$ th-order space harmonic for $n=$ $0, \pm 1, \pm 2, \ldots$. The phase constant of the $n$th space harmonic is displaced by $\pm\left(2 \pi / d_{2}\right) n$ from the fundamental space harmonic of order zero. $\beta_{p, 0}^{+}$and $\beta_{p, 0}^{-}$denote the phase constants of the corresponding forward and backward traveling unperturbed $\mathrm{EH}_{0}$ mode $(p=0)$ and $\mathrm{EH}_{1}$ mode $(p=1)$ of the guiding structure. In (1), $J_{0,0}^{+}$and $J_{1,0}^{+}$components denote the current amplitudes of the fundamental dominant $\left(\mathrm{EH}_{0}\right)$ and higher order $\left(\mathrm{EH}_{1}\right)$ forward traveling waves (both with positive phase velocity) of order zero, respectively. $J_{0, n}^{+}\left(J_{1, n}^{+}\right)$denotes the current amplitude of the $n$th higher order space harmonic that is associated with the fundamental dominant (higher order $\mathrm{EH}_{1}$ ) forward traveling wave. Contrarily, the current amplitudes with negative superscripts represent the $n$th higher order space harmonic that is subject to the fundamental dominant or higher order backward traveling wave.

The matrix-pencil method [18] can be applied to analyze the surface currents on the periodic structure as (1) assumes that the current distributions along the leaky line are of the exponential waveforms along the longitudinal axis (the $y$ direction). Therefore, the following expression is a valid approximation to the surface current $\boldsymbol{J}_{\mathrm{s}}$ :

$$
\boldsymbol{J}_{s}(x, y) \approx \sum_{i=1}^{M} \boldsymbol{J}_{i}(x) \exp \left(-j \gamma_{i} y\right) \quad M=1,2,3, \ldots
$$

where $\boldsymbol{J}_{s}(x, y)$ is a known quantity and is obtained by solving the periodic structure as shown in Fig. 2 [15]. $\gamma_{i}$ is the extracted complex propagation constant of the traveling wave component without prior knowledge of the dominant mode $\left(\mathrm{EH}_{0}\right)$, the higher-order mode $\left(\mathrm{EH}_{1}\right)$, their space harmonics, or other exponential waveforms. The integer $M$ in (3) represents the number of traveling waves applied to approximate the currents on the periodic structure. $\boldsymbol{J}_{\boldsymbol{i}}(x)$ is the extracted current amplitude of the $i$ th traveling wave component at location $x$ in the transverse plane. The matrix-pencil analyses of $\boldsymbol{J}_{s}$, sampled by taking sufficient points per wavelength along the $y$ axis, should yield (almost) the same propagation constant at various transverse locations of $x$. Extensive numerical studies of the particular guiding structure show that 12 sampling points per guided wavelength $\left(\lambda_{\mathrm{g}}\right)$ in the y direction, nine equally spaced locations in the $x$ direction, and 10 traveling components $(M=10)$ will yield a reasonably accurate estimate of the waves extracted from the periodic structure. The values of the complex propagation constants, $\gamma_{i}$, are nearly identical to within $1 \%$ accuracy, regardless of which of the nine transverse locations is considered.

The dispersion curves of the $\mathrm{EH}_{0}$ and $\mathrm{EH}_{1}$ modes displayed in Fig. 3 can be expressed in another form, with the phase constants as the abscissa and the free-space wavenumber $\mathrm{k}_{0}$ as the ordinate, and multiplying both by a constant, $\mathrm{d}_{2} / 2 \pi$. The plot is the familiar Brillouin diagram [16], [17], which is useful for understanding the propagation characteristics of the periodic structures. The dominant $\mathrm{EH}_{0}$ mode, for instance, has a normalized phase constant very close to 1.55 in the entire frequency band. 


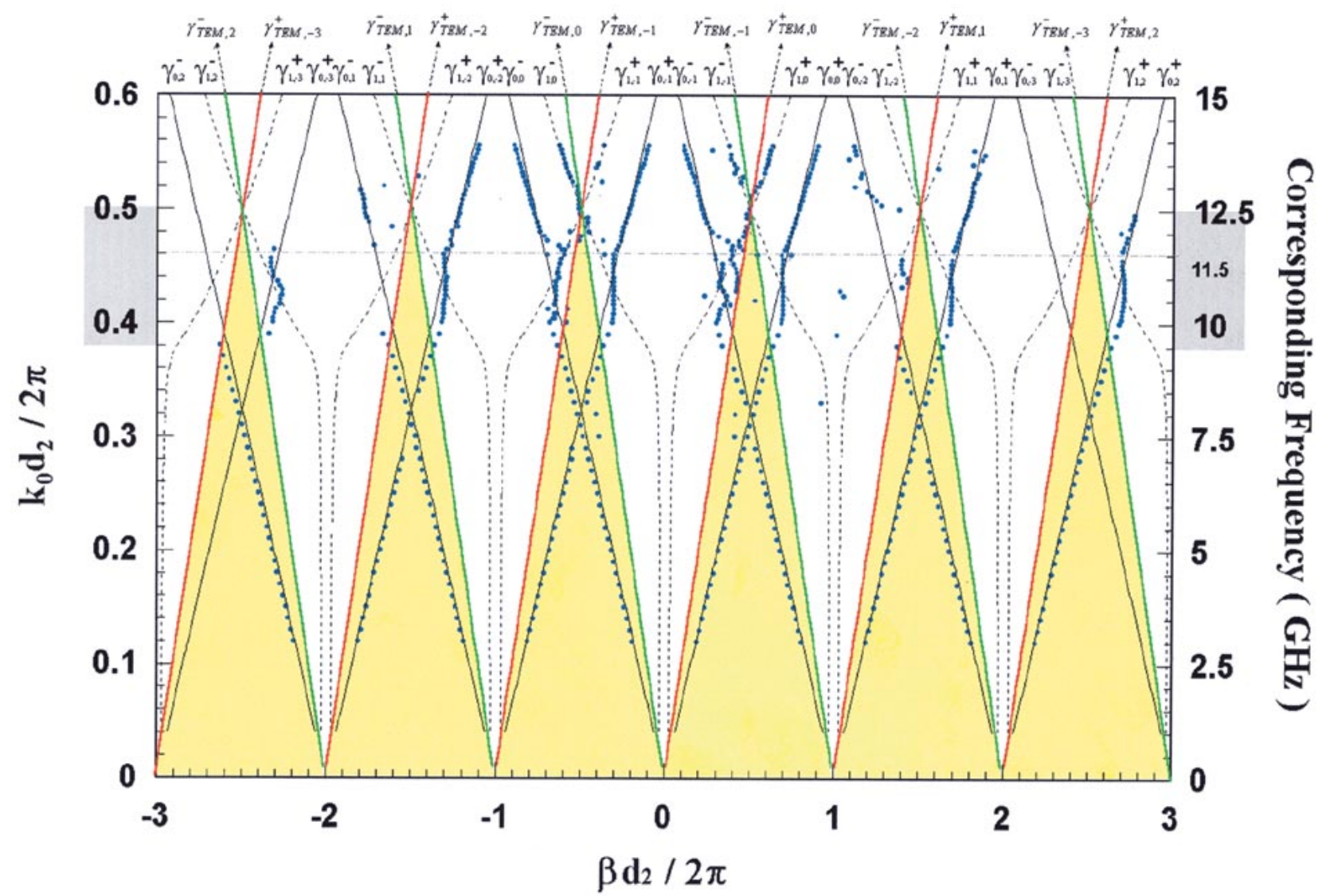

Fig. 6. Extracted phase constants $\left(\beta / \mathrm{k}_{0}\right)$ of the single leaky line, employing the matrix-pencil method with ten traveling components $(M=10)$ and superimposed onto the Brillouin diagram. The grayed frequency region is the leaky region of the first higher-order $\mathrm{mode}\left(\mathrm{EH}_{1}\right)$ from $9.4 \mathrm{GHz}$ $\left(\mathrm{k}_{0} \mathrm{~d}_{2} / 2 \pi=0.376\right)$ to $12.5 \mathrm{GHz}\left(\mathrm{k}_{0} \mathrm{~d}_{2} / 2 \pi=0.5\right)$.

Thus, the dispersion curve of the $\mathrm{EH}_{0}$ mode, when displayed in the Brillouin diagram, will be an almost straight line, beginning from the origin of the abscissa $\left(\beta \mathrm{d}_{2} / 2 \pi\right)$ with a slope 0.645 $(1 / 1.55)$, as the solid, black curve labeled $\gamma_{0,0}^{+}$in Fig. 6. However, the normalized phase constant of the higher-order $\mathrm{EH}_{1}$ mode, as depicted in Fig. 3, is a dashed, black curve, $\gamma_{1,0}^{+}$, in the Brillouin diagram (Fig. 6). The dispersion curve of the $n$th space harmonic is displaced by $\pm n$ from the fundamental space harmonic in the Brillouin diagram. The top of Fig. 6 is labeled as the appropriate representations of the space harmonics $\gamma_{0, n}^{ \pm}$and $\gamma_{1, n}^{ \pm}$derived from the dominant $\left(\mathrm{EH}_{0}\right)$ and first higher-order $\left(\mathrm{EH}_{1}\right)$ modes of order $n(n=0, \pm 1, \pm 2, \ldots)$, respectively.

The propagation characteristics of space harmonics, $\gamma_{0, n}^{+}$, $\gamma_{1, n}^{+}, \gamma_{0, n}^{-}$, and $\gamma_{1, n}^{-}$are specified as [19].

Forward traveling waves (FTW, $\beta>0$ ): $\gamma_{0, n}^{+}, \gamma_{1, n}^{+}$, if $n \geq$ 0 , and $\gamma_{0, n}^{-}, \gamma_{1, n}^{-}$, if $n<0$.

Backward traveling waves (BTW, $\beta<0$ ): $\gamma_{0, n}^{+}, \gamma_{1, n}^{+}$, if $n<0$, and $\gamma_{0, n}^{-}, \gamma_{1, n}^{-}$, if $n \geq 0$.

Forward waves (FW, $\left.\beta \cdot(\partial \beta / \partial \omega)^{-1}>0\right): \gamma_{0, n}^{+}, \gamma_{1, n}^{+}, \gamma_{0, n}^{-}$, and $\gamma_{1, n}^{-}$, if $n \geq 0$.

Backward waves (BW, $\left.\beta \cdot(\partial \beta / \partial \omega)^{-1}<0\right): \gamma_{0, n}^{+}, \gamma_{1, n}^{+}$, $\gamma_{0, n}^{-}$, and $\gamma_{1, n}^{-}$, if $n<0$.

Following the previous notion, a family of curves, $\gamma_{0, n}^{+}\left(\gamma_{0, n}^{-}\right)$, are plotted at distance $n(-n)$ from $\gamma_{0,0}^{+}\left(\gamma_{0,0}^{-}\right)$in the Brillouin diagram. Similarly $\gamma_{1, n}^{+}\left(\gamma_{1, n}^{-}\right)$is shifted by $n(-n)$ from $\gamma_{1,0}^{+}$ $\left(\gamma_{1,0}^{-}\right)$for the $n$ th-order space harmonic. The dispersion curves of the space harmonics of the unloaded air-filled TEM lines is plotted to complete the background information on various types of space harmonics. Straight lines of slope $+1(-1)$ are plotted in red (green) and labeled as $\gamma_{T E M, n}^{+}\left(\gamma_{T E M, n}^{-}\right)$where $n=0, \pm 1, \pm 2, \ldots$. Notably, the points of intersection of the red and green lines are at $((2 n+1) / 2,0.5)$. The intersects of these straight lines form an infinite array of (yellow) triangles. The waves are bound inside the triangles; however, outside the triangles is the radiation region. The space harmonics in the radiation region are leaky waves. At the onset frequency $(12.5 \mathrm{GHz})$ of the higher-order $\mathrm{EH}_{1}$ mode, the $\gamma_{1,0}^{+}$curve must cross the straight line defined by $\beta / \mathrm{k}_{0}=1$. Therefore, in the Brillouin diagram, the space harmonics of $\gamma_{1,0}^{+}\left(\right.$or $\left.\gamma_{1,0}^{-}\right)$intersect the TEM lines. The points of intersection must be at 0.5 of the ordinate $\left(\mathrm{k}_{0} \mathrm{~d}_{2} / 2 \pi\right)$ since the periodicity, $\mathrm{d}_{2}(12.0 \mathrm{~mm})$, of the perturbations is chosen to be one half of the free-space wavelength $(24.0 \mathrm{~mm})$ at the onset frequency.

The aforementioned space harmonics and triangles, thus, constitute a template for understanding the propagation characteristics of the periodic leaky line. The phase constants (real parts) of the extracted $\gamma_{i}$ in (3) are also represented in the Brillouin diagram as the dotted symbols in Fig. 6. Fig. 6 reveals that the extracted complex propagation constants in the Brillouin diagram fall near the solid and dashed curves of the template except at a few locations. That is, the extracted solutions nearly coincide with the theoretical space harmonics $\gamma_{0, n}^{ \pm}$and $\gamma_{1, n}^{ \pm}$verifying the assumption in (1) and (2), that the 
TABLE I

EXTRACTED COMPONENTS OF VARIOUS WAVES OF Fig. 2 AT $11.5 \mathrm{GHz}\left(\mathrm{k}_{0} \mathrm{~d}_{2} / 2 \pi=0.46\right)$

\begin{tabular}{|c|c|c|c|c|c|c|c|c|c|c|}
\hline $\begin{array}{c}\text { Order of } \\
\text { Eq. (3) }\end{array}$ & $\begin{array}{l}\text { ith space } \\
\text { harmonic }\end{array}$ & \begin{tabular}{|c|} 
Normalized \\
phase \\
constant \\
\end{tabular} & \begin{tabular}{|c|}
$\begin{array}{c}\text { Normalized } \\
\text { attenuation } \\
\text { constant }\end{array}$ \\
\end{tabular} & \begin{tabular}{|c|} 
Normalized \\
current \\
amplitude \\
\end{tabular} & $\begin{array}{c}\text { Beam angle } \\
\text { of leaky wave }\end{array}$ & $\begin{array}{c}\text { Corresponding } \\
\text { space harmonics of } \\
\text { Brillouin diagram } \\
\end{array}$ & \multicolumn{4}{|c|}{$\begin{array}{l}\text { Propagation characteristics of } \\
\text { space harmonics }\end{array}$} \\
\hline \multirow{2}{*}{$M$} & \multirow{2}{*}{$i$} & \multirow{2}{*}{$\beta / k_{0}$} & \multirow{2}{*}{$\alpha / k_{0}$} & \multirow{2}{*}{$\left(\overline{I_{i}}\right)$} & \multirow{2}{*}{$\theta=\sin ^{-1}\left(\beta / k_{0}\right)$} & \multirow{2}{*}{$\gamma_{m, n}^{ \pm}$} & \multicolumn{4}{|c|}{ Types } \\
\hline & & & & & & & $F T W$ & $F W$ & $B T W$ & $B W$ \\
\hline \multirow{10}{*}{$M=10$} & 1 & 1.484 & 0.065 & 1 & $* * * * *$ & $\gamma_{0,0}^{+}$ & $\sqrt{ }$ & $\sqrt{ }$ & & \\
\hline & 2 & -0.67 & 0.106 & 0.65 & $-42.093^{\circ}$ & $\gamma_{0,-1}^{+}$ & & & $\sqrt{ }$ & $\sqrt{ }$ \\
\hline & 3 & -1.303 & 0.149 & 0.418 & $* * * * *$ & $\gamma_{1,-1}^{+}$ & & & $\sqrt{ }$ & $\sqrt{ }$ \\
\hline & 4 & 0.858 & 0.098 & 0.304 & $59.116^{\circ}$ & $\gamma_{1,0}^{+}$ & $\sqrt{ }$ & $\sqrt{ }$ & & \\
\hline & 5 & 1.606 & 0.066 & 0.287 & $* * * * *$ & $\gamma_{0,0}^{+}$ & $\sqrt{ }$ & $\sqrt{ }$ & & \\
\hline & 6 & -2.848 & 0.099 & 0.21 & $* * * * *$ & $\gamma_{0,-2}^{+}$ & & & $\sqrt{ }$ & $\sqrt{ }$ \\
\hline & 7 & -0.79 & 0.01 & 0.074 & $-52.164^{\circ}$ & $\gamma_{1,0}^{-}$ & & $\sqrt{ }$ & $\sqrt{ }$ & \\
\hline & 8 & 0.632 & 0.061 & 0.051 & $39.173^{\circ}$ & $\gamma_{0,-1}^{-}$ & $\sqrt{ }$ & & & $\sqrt{ }$ \\
\hline & 9 & -1.524 & -0.065 & 0.006 & $* * * * *$ & $\gamma_{0,0}^{-}$ & & $\sqrt{ }$ & $\sqrt{ }$ & \\
\hline & 10 & 1.171 & -0.081 & 0.003 & $* * * * *$ & $\gamma_{1,-1}^{-}$ & $\sqrt{ }$ & & & $\sqrt{ }$ \\
\hline
\end{tabular}

$\mathrm{EH}_{0}$ and $\mathrm{EH}_{1}$ modes and their higher-order space harmonics approximate closely the current distributions of the leaky line.

Table I specifies the extracted traveling components at $11.5 \mathrm{GHz}$, assuming $M=10$, determined by the matrix-pencil method, to supplement the loss of information about the amplitudes of the space harmonics. The third and fourth columns of Table I list the extracted normalized phase constant and attenuation constant, and the fifth column gives the relative current amplitudes. The normalized current amplitude is the ratio of the magnitude of the total current of the $i$ th traveling wave component to the largest total current magnitude. Therefore, the normalized current amplitude $\bar{I} i$ in the matrix-pencil method can be given by

$$
\begin{aligned}
I_{i} & \approx \sum_{j=1}^{N}\left|\boldsymbol{J}_{i}\left(x_{j}\right)\right| \cdot\left|\Delta x_{j}\right| \\
\bar{I}_{i} & =\frac{I_{i}}{\left(I_{i}\right)_{\operatorname{Max}}} .
\end{aligned}
$$

$N$ equals nine as explained earlier. Table I specifies the $i$ th traveling wave component, according to the descending normalized current amplitude. The smallest relative current amplitude is approximately $0.3 \%$ of the largest component, in contrast to $2.5 \%$, $17.4 \%$, and $22.6 \%$ for $M=8,6$, and 4, respectively. Clearly, the series representation of the surface current, $J_{\mathrm{s}}$, as in (3), quickly converges as $M$ increases; $M=10$ appear to be a good approximation for extracting the traveling wave components in the particular case study. A traveling wave with $\left|\beta / k_{0}\right|$ below unity, must be outside the triangles of the Brillouin diagram and thus is a leaky wave. The beam angle of this leaky wave points to the positive (negative) $y$ axis if $0<\beta / k_{0}<1\left(-1<\beta / k_{0}<0\right.$ ). The beam angle of the leaky wave from the broadside is approximately

$$
\theta=\sin ^{-1}\left(\frac{\beta}{k_{0}}\right) \text { for }\left|\frac{\beta}{k_{0}}\right|<1 .
$$

The seventh column of Table I denotes the corresponding space harmonics as seen in the Brillouin diagram of Fig. 6. The types of the space harmonics are identified in the rightmost (eighth) column of Table I. The fundamental $\gamma_{0,0}^{+}$has the largest current component ( $i=1$ in Table I). The backward leaky waves $\gamma_{0,-1}^{+}(i=2)$ and $\gamma_{1,-1}^{+}(i=3)$ have the second and third largest current components, respectively. $\gamma_{1,0}^{+}$makes the fourth largest contribution to the excited currents of the leaky line $(i=4)$, and so on. The beam angles in the sixth column were determined by (5). Importantly, two leaky space waves contribute primarily radiation, $\gamma_{0,-1}^{+}(i=2)$ and $\gamma_{1,0}^{+}(i=4)$. Furthermore, as stated earlier, the radiation region is outside the triangles bounded by the air-filled TEM waves (red and green lines in Fig. 6). Consequently, the space harmonics beyond the onset frequency, $12.5 \mathrm{GHz}$, of the $\mathrm{EH}_{1}$ mode are in the radiation region, thus producing radiation loss beyond $12.5 \mathrm{GHz}$ as the significant return loss with $\left|\mathrm{S}_{11}\right|$ and RPA values as predicted in Figs. 4 and 5, respectively. Subsequently, most space harmonics in the frequency band from 9.4 to $12.5 \mathrm{GHz}$ (leaky region of the $\mathrm{EH}_{1}$ mode) also fall into the radiation region (outside the triangles) and thus radiate energy. These radiation characteristics explain why the frequency band above $9.4 \mathrm{GHz}$ dominates the radiation losses, as discussed in Section II. However, in the frequency band below $9.4 \mathrm{GHz}$, almost all space harmonics fall inside the triangles and are bound.

Further investigating the current distribution $\boldsymbol{J}_{i}\left(x_{j}\right)$ in the transverse plane of the various space harmonics reveals that the symmetry of a specific wave will be altered by the presence of antisymmetric perturbations. For example, the longitudinal current component of $\gamma_{0,0}^{+}$is evenly symmetric; however, the longitudinal current component of the higher-order space harmonic of order $-1\left(\gamma_{0,-1}^{+}\right)$is oddly symmetric. Similarly, the longitudinal current component of $\gamma_{1,0}^{+}$is oddly symmetric but its space harmonic $\gamma_{1,-1}^{+}$shows an evenly symmetric current distribution. The oddly symmetric $\gamma_{0,-1}^{+}$and $\gamma_{1,0}^{+}$components are 


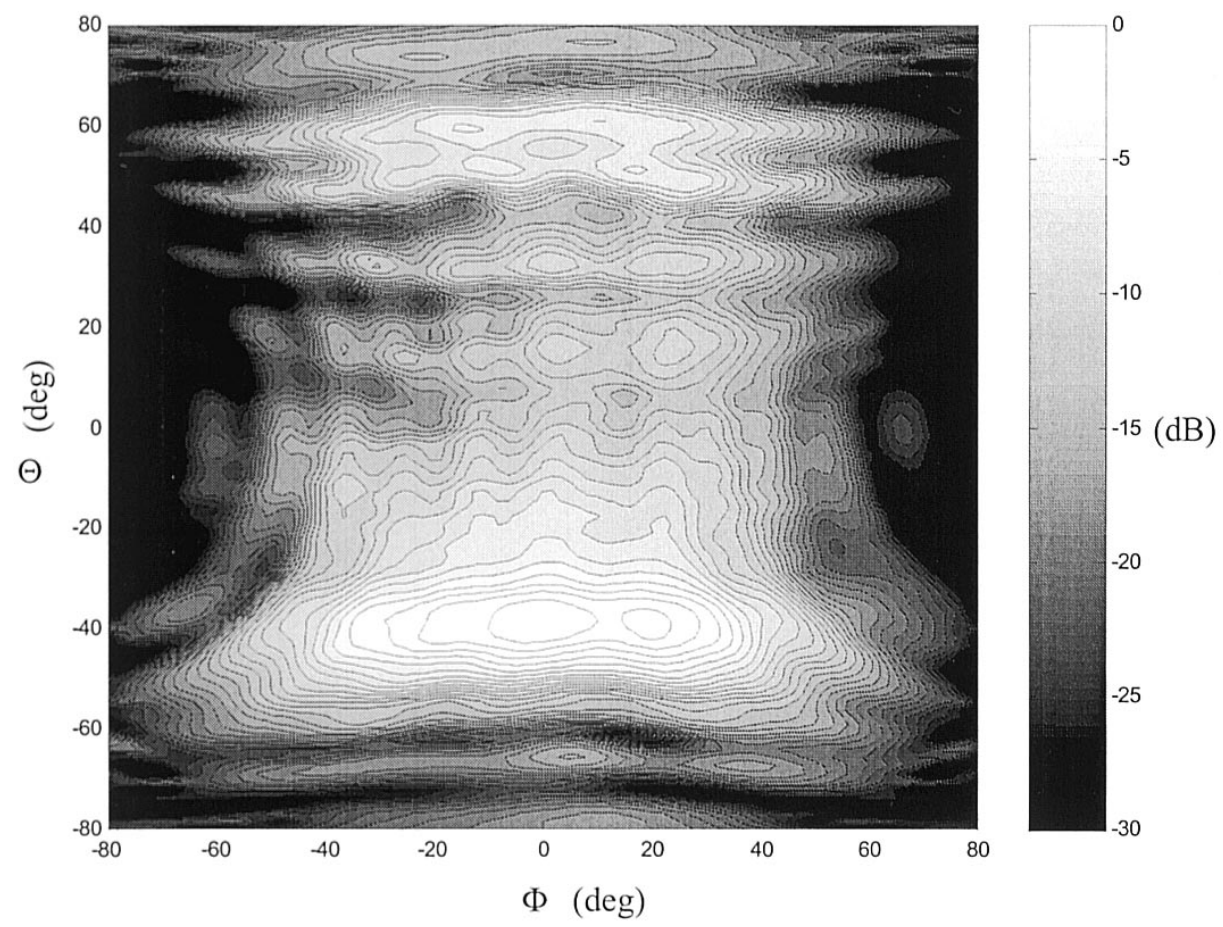

Fig. 7. Measured far-field radiation contour $\left(\mathrm{E}_{\phi}\right)$ of the single leaky-line prototype (Fig. 2) at $11.5 \mathrm{GHz}$ (contour step $=1 \mathrm{~dB}$ ).

outside the triangles and lie in the backward and forward leaky regions, respectively. Accordingly, both of these two forward and backward leaky waves of a single leaky line have the same polarization but differ in amplitude (see Fig. 1).

\section{RADIATION CHARACTERISTICS OF THE SingLE LEAKy LiNE AND RADIAL ANTENNA ARRAY}

\section{A. Single Leaky Line}

This section relates the radiation beam angles predicted by the space harmonics, as listed in Table I, to those obtained by measurement. The far-field radiation patterns, obtained by the near-field measurement system, are expressed by $\Phi$ and $\Theta$ [20]

$$
\begin{aligned}
& \Phi=\tan ^{-1}[\tan (\theta) \cos (\phi)]=\tan ^{-1}\left(\frac{K_{x}}{K_{z}}\right) \\
& \Theta=\sin ^{-1}[\sin (\theta) \sin (\phi)]=\sin ^{-1}\left(K_{y}\right) .
\end{aligned}
$$

$\theta$ and $\phi$, in (6) and (7), are the angle parameters in spherical coordinates. In the near-field measurement system, $K_{x}$ and $K_{z}$ are the horizontal and depth components in the K-space domain, respectively. $K_{y}$ is the vertical component. $K_{x}, K_{y}$, and $K_{z}$ can be defined in a spherical coordinate system

$$
\begin{aligned}
K_{x} & =\sin \theta \cos \phi \\
K_{y} & =\sin \theta \sin \phi \\
K_{z} & =\cos \theta .
\end{aligned}
$$

When measuring an antenna in the near-field measurement system, we set the $y$ axis of the antenna in the vertical direction. The $x$ axis and $z$ axis are in the horizontal and depth directions, respectively. Therefore, $\phi=90^{\circ}\left(\theta=0^{\circ}\right)$ corresponds to $\Phi=0^{\circ}\left(\Theta=0^{\circ}\right.$ and $\left.\Phi=0^{\circ}\right)$. The polarization of the

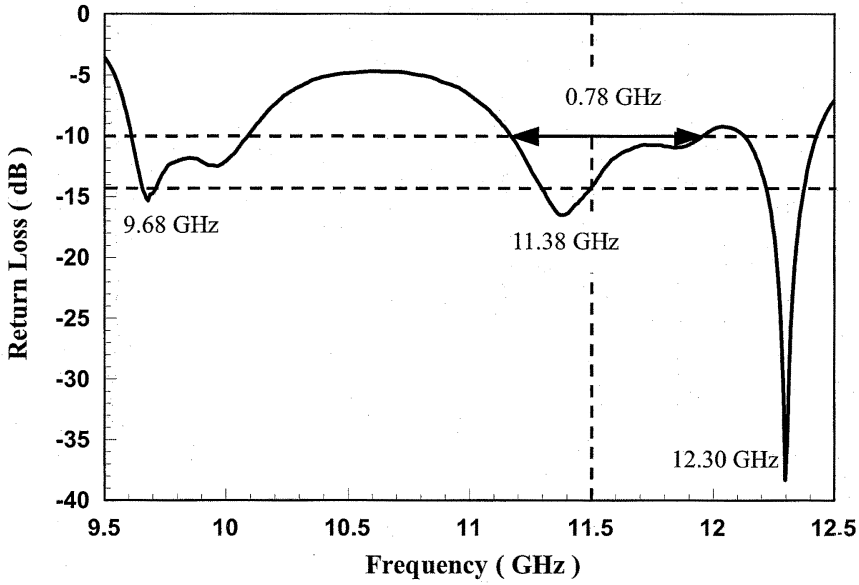

Fig. 8. Measured return loss $\left(\left|\mathrm{S}_{11}\right|\right)$ of the proposed microstrip radial antenna array.

waveguide probe at the scanner should coincide with the polarization of the antenna under test (AUT) for copolarization measurement. A positive (negative) $\Theta$ of the radiation beam represents forward (backward) radiation in the $+y(-y)$ direction. The theoretical results in Table I indicate that, with amplitudes in descending order, the first and second beams point at $\Theta$ equal to $-42^{\circ}$ and $+59^{\circ}$ at $\Phi=0^{\circ}$, respectively. The main beam at $-42^{\circ}$ is associated with the backward leaky wave, $\gamma_{0,-1}^{+}$, and the second beam with the forward leaky wave $\gamma_{1,0}^{+}$. However, the sidelobes contributed by the space harmonics $\gamma_{1,0}^{-}$and $\gamma_{0,-1}^{-}$are not obvious because they have small amplitudes, as extracted by the matrix-pencil method. An open-ended leaky-line prototype is built and tested according with the dimensions and material constant given in Fig. 2. The predominant $\mathrm{E}_{\phi}$ component of the leaky line manifests the horizontal polarization in the near-field measurement system. Thus, $y-z$ plane is the $\mathrm{H}$ plane. Fig. 7 


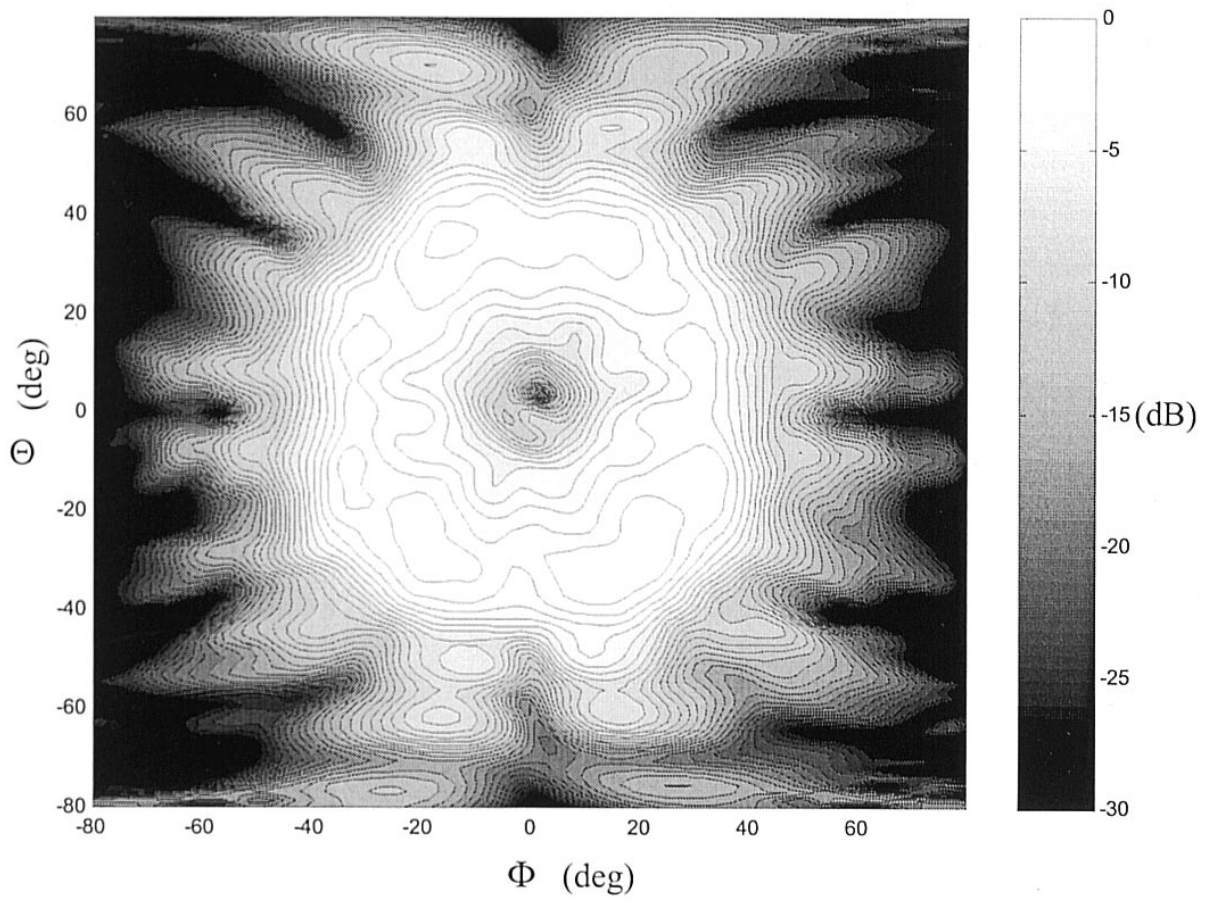

Fig. 9. Measured conical beam (in $\mathrm{dB}$ ) of the proposed microstrip radial antenna array at $11.5 \mathrm{GHz}$. (contour step $=1 \mathrm{~dB}$ ).

plots the measured radiation contours, which show that the main beam points to $-40^{\circ}$ and the second beam to $+60^{\circ}$ in $\Theta$ (at $\Phi=0^{\circ}$ ). Accordingly, the theory presented in the preceding section agrees closely with the experimental results. The measured directivity is $15.88 \mathrm{dBi}$ and its 3 - $\mathrm{dB}$ (half-power) contour spans $64^{\circ}$ in $\Phi$ and $15^{\circ}$ in $\Theta$. (See the lower part of Fig. 7.) Such an advantageous radiation pattern is applied for a low-profile microstrip antenna array to construct a conical beam, since the main beam is thin in $\Theta$ and wide in $\Phi$.

\section{B. Radial Antenna Array}

In view of the requirement for a conical-beam antenna, eight leaky lines are applied to synthesize an antenna array with this feature. Fig. 1 illustrates the basic operational principle of the radial antenna array: each leaky line radiates a main beam in the backward direction and a second beam in the forward direction. Both beams have approximately equal 3-dB beamwidths in $\Phi$, as depicted in Fig. 7. Although both beams have different $\Theta$ angles, maintaining the colinear arrangement of the two opposite leaky lines, as indicated in Fig. 1, ensures that the conical beam is obtained and its 3-dB beamwidth widened. In practice, a shorter leaky line than that described in the previous section is used to shrink the antenna array, while maintaining the conical beam. Therefore, a leaky line, possessing seven periods instead of the fourteen periods of the leaky-line prototype with antisymmetric square holes on the ground plane, is employed to form an eight-element radial antenna array. The eight elements are equally spaced around a circle, as depicted in Fig. 1. An SMA connector is directly mounted on the backside of the antenna array and its central pin is soldered to the center of the front side. Fig. 8 shows that the measured input return losses are -15.3 , -16.5 , and $-38.3 \mathrm{~dB}$ at $9.68,11.38$, and $12.30 \mathrm{GHz}$, respectively, indicating good $50-\Omega$ input matching. The return loss is

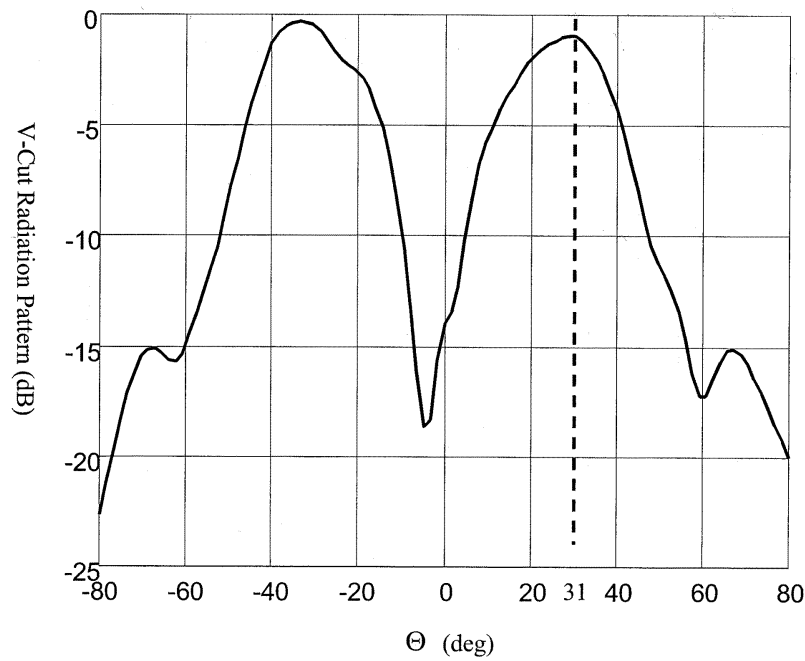

Fig. 10. The vertical cut (V-cut, $\Phi=0^{\circ}$ ) of the measured conical beam in Fig. 9.

$-14.3 \mathrm{~dB}$ at the $11.5-\mathrm{GHz}$ operating frequency, also showing good matching characteristics. The bandwidth as defined by $\left|\mathrm{S}_{11}\right|<-10 \mathrm{~dB}$, is from 11.17 to $11.95 \mathrm{GHz}$, or approximately $6.8 \%$. The polarization of Fig. 7 (single leaky line) is horizontal and thus the radial antenna array has vertical and horizontal polarizations simultaneously. Accordingly, we should measure the radial antenna array twice for the two different polarizations and then superpose the two far-field results to obtain the total field pattern. Fig. 9 depicts the measured radiation pattern of the radial antenna array at $11.5 \mathrm{GHz}$, approximately showing a circular fashion in $\Theta-\Phi$ plane. The circular pattern in Fig. 9 indicates a conical beam in spherical coordinates with a flare angle approximately equal to the radius of the circle. Thus the flare angle of the conical beam shown in Fig. 9 is $31^{\circ}$. The detailed 


$$
\begin{aligned}
& \left\{\tan ^{-1}[\tan (\theta) \cos (\phi)]\right\}^{2}+\left\{\sin ^{-1}[\sin (\theta) \sin (\phi)]\right\}^{2} \sim \theta^{2} \\
& \text { or } \Phi^{2}+\Theta^{2} \sim \theta^{2} \text { if } \theta<0.7(\operatorname{rad}) \text { or } \theta<40^{\circ}, \text { with } \\
& \frac{\operatorname{Max}\left(\sqrt{\left\{\tan ^{-1}[\tan (\theta) \cos (\phi)]\right\}^{2}+\left\{\sin ^{-1}[\sin (\theta) \sin (\phi)]\right\}^{2}}\right)-\theta}{\theta}<2.3 \%
\end{aligned}
$$

expressions are in the Appendix. The relative power level at the center of the conical beam is about $-18 \mathrm{~dB}$. Fig. 10 shows the vertical cut (V-cut) of the conical beam at $\Phi=0^{\circ}$ for clearer illustration of radius $\theta$. As seen in Fig. 10, the main beam of the conical beam has a flare angle of $31^{\circ}$ and has a $3-\mathrm{dB}$ beamwidth of $24^{\circ}$. The microstrip radial antenna array with a conical beam can be applied for an indoor WLAN, mobile, and satellite applications.

\section{CONCLUSION}

This paper shows the controllability of leaky waves in the form of space harmonics, by introducing perturbations of the ground plane-namely, antisymmetric etched holes. The original $\mathrm{EH}_{0}$ and $\mathrm{EH}_{1}$ modes supported by the otherwise ideal microstrip experience the space-harmonic modulations by the perturbations from the ground plane. The space harmonics $\gamma_{0,-1}^{+}$ and $\gamma_{1,0}^{+}$of the leaky-line prototype are shown to contribute the main beam and second beam, respectively. The tilt angles of the measured main beam and second beam closely correspond to the theoretical prediction derived from the Brillouin diagram of the leaky line. Measurement of an eight-element radial antenna array formed by four pairs of identical, colinear leaky lines shows a conical beam. The conical beam has a flare angle of $31^{\circ}$ and a $3-\mathrm{dB}$ beamwidth of $24^{\circ}$ at $11.5 \mathrm{GHz}$. Such a pattern can be used for indoor WLAN, mobile, and satellite communications.

\section{APPENDIX}

By (6) and (7), numerical studies show that conical beams in spherical coordinates with smaller flare angles $\theta(\theta<0.7(\mathrm{rad})$ or $\theta<40^{\circ}$ ) nearly maps to circles with radii $\theta$ in $\Theta-\Phi$ plane with maximum discrepancies less than $2.3 \%$. That is (see (A1) and (A2) at the top of the page).

The radii $\theta$ in (A1) and (A2) are presented by radians and are converted to degrees shown in Fig. 9. Therefore, the pattern shown in Fig. 9 is a conical beam with a flare angle equal to $\theta\left(\theta=31^{\circ}\right)$.

\section{ACKNOWLEDGMENT}

Authors wish to extend their appreciations to Prof. Tapan Kumar Sarkar for his kind supervisions of matrix-pencil codes as well as to Mr. Yu-Chiao Chen for assisting data presentations.

\section{REFERENCES}

[1] "Mini-special issue on electromagnetic crystal structures, design, synthesis and applications," IEEE Trans. Microwave Theory Tech., vol. 47, pp. 2059-2166, Nov. 1999.
[2] V. Radisic, Y. Qian, R. Coccioli, and T. Itoh, "Novel 2-D photonic bandgap structure for microstrip lines," IEEE Microwave Guided Wave Lett., vol. 8, pp. 69-71, Feb. 1998.

[3] K. C. Chen, C. K. C. Tzuang, Y. Qian, and T. Itoh, "Leaky properties of microstrip above a perforated ground plane," in IEEE MTT-S Int. Microwave Symp. Dig., 1999, pp. 69-72.

[4] A. Petosa and J. S. Wight, "Analysis of a low profile slotted microstrip antenna for cellular mobile radio," IEEE Trans. Veh. Technol., vol. 41, pp. 363-368, Nov. 1992.

[5] K. Fujimoto, T. Hori, S. Nishimura, and K. Hirasawa, "Applications in mobile and satellite systems," in Handbook of Microstrip Antennas, Inst. Elect. Eng. Electromagnetic Waves Series 28, J. R. James and P. S. Hall, Eds., 1989, vol. 2, ch. 19, pp. 1127-1132.

[6] W. Menzel, "A new travelling-wave antenna in microstrip," AEÜ 33, pp. 137-140, Apr. 1979.

[7] G. J. Chou and C. K. C. Tzuang, "An integrated quasiplanar leaky-wave antenna," IEEE Trans. Antennas Propagat., vol. 44, pp. 1078-1085, Aug. 1996.

[8] Y. D. Lin, J. W. Sheen, and C. K. C. Tzuang, "Analysis and design of feeding structures for microstrip leaky wave antenna," IEEE Trans. Microwave Theory Tech., vol. 44, pp. 1540-1547, Sept. 1996.

[9] C. N. Hu and C. K. C. Tzuang, "Microstrip leaky-mode antenna array," IEEE Trans. Antennas Propagat., vol. 45, pp. 1698-1699, Nov. 1997.

[10] T. L. Chen and Y. D. Lin, "Aperture-coupled microstrip line leaky wave antenna with broadside mainbeam," Electron. Lett., vol. 34, pp. 1366-1367, July 1998.

[11] Y. Qian, B. C. C. Chang, T. Itoh, K. C. Chen, and C. K. C. Tzuang, "High efficiency and broadband excitation of leaky mode in microstrip structures," in IEEE MTT-S Int. Microwave Symp. Dig., 1999, pp. $1419-1422$.

[12] C. C. Lin and C. K. C. Tzuang, "A dual-beam micro-CPW leaky-mode antenna," IEEE Trans. Antennas Propagat., pp. 310-316, Feb. 2000.

[13] M. Cohn, B. D. Geller, and J. M. Schellenberg, "A 10 watt broadband FET combiner/amplifier," in IEEE MTT-S Int. Microwave Symp. Dig., 1979, pp. 292-297.

[14] K. S. Lee, "Microstrip line leaky wave antenna," Ph.D. dissertation, Rensselaer Polytech. Inst. New York, June 1986.

[15] Zeland IE3D Version 8.0, Zeland Software, Inc..

[16] A. A. Oliner, Radiating periodic structures: analysis in terms of $k$ vs. $\beta$ diagrams, in SHORT COURSE on Microwave Field and Network Techniques, June 4, 1963

[17] R. E. Collin and F. J. Zucker, Antenna Theory. New York: McGrawHill, 1969, pt. 2, ch. 19.

[18] R. S. Adve, T. K. Sarkar, O. M. C. Pereira-Filho, and S. M. Rao, "Extrapolation of time-domain responses from three-dimensional conducting objects utilizing the matrix pencil technique," IEEE Trans. Antennas Propagat., vol. 45, pp. 147-156, Jan. 1997.

[19] C. K. C. Tzuang and J. M. Lin, "On the mode-coupling formation of complex modes in a nonreciprocal finline," IEEE Trans. Microwave Theory Tech., vol. 41, pp. 1400-1408, Aug. 1993.

[20] D. Slater, Near-Field Antenna Measurements. Norwood, MA: Artech House, 1991, ch. 3, pp. 50-51.

Kuo-Cheng Chen was born in Taiwan, R.O.C., on December 26, 1972. He received the B.S. and Ph.D. degrees in electrical communication engineering from the National Chiao Tung University (NCTU), Hsinchu, Taiwan, R.O.C., in 1994 and 2002 respectively. His research activities involve the design and development of (periodical) leaky-wave antenna arrays, reflect arrays, and microwave passive circuits. 
Yongxi Qian (S'09-M'93-SM'00) was born in Shanghai, China, in 1965. He received the B.E. degree from Tsinghua University, Beijing, in 1987 and the M.E. and Ph.D. degrees from the University of Electro-Communications, Tokyo, Japan, in 1990 and 1993, respectively, all in electrical engineering.

From 1993 to 1996, he was an Assistant Professor with the University of Electro-Communications. From April 1996 to January 2001, he was a Postdoctoral Fellow, Assistant Research Engineer, and Lecturer with the Electrical Engineering Department, University of California, Los Angeles (UCLA). His research interests included numerical techniques for microwave and millimeter-wave circuits and antennas, generation and transmission of picosecond electrical pulses, crosstalk problems in high-density MMICs, miniature circuits for mobile communications, $60-\mathrm{GHz}$ millimeter-wave focal plane imaging array, broadband planar antennas, smart antennas and arrays for wireless communications, high-efficiency microwave power amplifiers, RF interconnect for mixed signal silicon MMICs, quasi-optical power combining, photonic band-gap (PBG) structures, active integrated antennas for indoor LANs, and high-power, broadband RF photonic devices for millimeter and submillimeter-wave photomixing. He has authored or coauthored more than 200 refereed journal and conference papers, two books, and several book chapters. He joined Microsemi Corporation in 2001 and has been working as Director of Technology and Applications at the MicroWaveSys Division focusing on advanced HBT ICs for $3 \mathrm{G}$, wireless LAN and high-speed fiber-optic applications.

Dr. Qian is the recipient of the Japan Microwave Prize at 1998 Asia-Pacific Microwave Conference, a Best Student Paper at 29th European Microwave Conference (1999), and the ISAP Paper Award at International Symposium on Antennas and Propagations (2000).

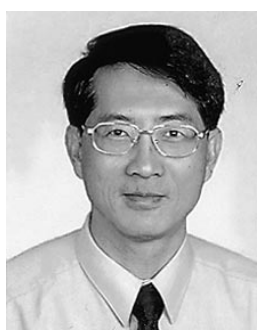

Ching-Kuang C. Tzuang (S'80-M'80-SM'92 -F'99) received the B.S. degree in electronic engineering from the National Chiao Tung University, Hsinchu, Taiwan, R.O.C., in 1977, the M.S. degree from the University of California at Los Angeles, in 1980, and the Ph.D. degree in electrical engineering from the University of Texas at Austin, in 1986.

From 1981 to 1984, he was with TRW, Redondo Beach, CA, where he was involved with analog and digital monolithic microwave integrated circuits. Since 1986, he has been with the Institute of Communication Engineering, National Chiao Tung University. His research activities involve the design and development of millimeter-wave and microwave active and passive circuits and the field theory analysis and design of various complex waveguiding structures and large-array antennas. He has supervised 58 M.Sc. and $18 \mathrm{Ph} . \mathrm{D}$. students.

Dr. Tzuang assisted in the formation of the IEEE Microwave Theory and Techniques Society (IEEE MTT-S), Taipei Chapter. He has served as secretary, vice chairman, and chairman in 1988, 1989, and 1990, respectively. Since 1994, he has also been on the Asia-Pacific Microwave Conference International Steering Committee, where he was the international liaison officer representing the Taipei Chapter. He received the Outstanding Engineering Professor Awards from the Chinese Institute of Electrical Engineering and the Chinese Institute of Engineering in 1999 and 200, respectively. He became an Acer-endowed Chair Professor in 2000.
Tatsuo Itoh (S'69-M'69-SM'74-F'82-LF'94) received the Ph.D. degree in electrical engineering from the University of Illinois, Urbana, in 1969.

From September 1966 to April 1976, he was with the Electrical Engineering Department, University of Illinois. From April 1976 to August 1977, he was a Senior Research Engineer in the Radio Physics Laboratory, SRI International, Menlo Park, CA. From August 1977 to June 1978, he was an Associate Professor with the University of Kentucky, Lexington. In July 1978, he joined the faculty of The University of Texas at Austin, where he became a Professor of electrical engineering in 1981 and Director of the Electrical Engineering Research Laboratory in 1984. During the summer of 1979, he was a guest researcher at AEG-Telefunken, Ulm, West Germany. In September 1983, he was selected to hold the Hayden Head Centennial Professorship of Engineering at The University of Texas. In September 1984, he was appointed Associate Chairman for Research and Planning of the Electrical and Computer Engineering Department at The University of Texas. In January 1991, he joined the University of California, Los Angeles as Professor of electrical engineering and holder of the TRW Endowed Chair in Microwave and Millimeter Wave Electronics. He was an Honorary Visiting Professor at Nanjing Institute of Technology, China, and with the Japan Defense Academy. In April 1994, he was appointed as Adjunct Research Officer for Communications Research Laboratory, Ministry of Post and Telecommunication, Japan. He currently holds Visiting Professorship at University of Leeds, U.K., and is an External Examiner of Graduate Program of City University of Hong Kong. He has 290 journal publications, 590 refereed conference presentations, and has written 30 books/book chapters in the area of microwaves, millimeter-waves, antennas, and numerical electromagnetics. He generated $50 \mathrm{Ph} . \mathrm{D}$. students.

Dr. Itoh received a number of awards including Shida Award from Japanese Ministry of Post and Telecommunications in 1998, Japan Microwave Prize in 1998, IEEE Third Millennium Medal in 2000, and IEEE MTT Distinguished Educator Award in 2000. He is a member of the Institute of Electronics and Communication Engineers of Japan, and Commissions B and D of USNC/URSI. He served as Editor of the IEEE TRANSACTIONS ON MiCROWAVE THEORY AND TECHNIQUES for 1983-1985. He serves on the Administrative Committee of IEEE Microwave Theory and Techniques Society. He was Vice President of the Microwave Theory and Techniques Society in 1989 and President in 1990. He was Editor-in-Chief of the IEEE MICROWAVE AND GUIDED WAVE LETTERS from 1991 through 1994. He was elected as an Honorary Life Member of MTT Society in 1994. He was the Chairman of USNC/URSI Commission D from 1988 to 1990, and Chairman of Commission D of the International URSI for 1993-1996. He is Chair of Long Range Planning Committee of URSI. He serves on advisory boards and committees of a number of organizations. 